\title{
3 MICRON IMAGING OF THE HUBBLE DEEP FIELD ${ }^{1}$
}

\author{
David W. Hogg, ${ }^{2,3}$ Gerry Neugebauer, ${ }^{4}$ Judith G. Cohen,${ }^{4}$ Mark Dickinson, ${ }^{5}$ S. G. Djorgovski, ${ }^{4}$ \\ KeIth MAtThews, ${ }^{4}$ AND B. T. SoIfER ${ }^{4}$ \\ Received 1999 October 27; accepted 2000 January 4
}

\begin{abstract}
Images of the Hubble Deep Field (HDF) at $3.2 \mu \mathrm{m}$, taken with the $10 \mathrm{~m}$ Keck Telescope, are presented. The images cover a total area of $\sim 2.5 \mathrm{arcmin}^{2}$. To a $5 \sigma$ limit of [3.2] $]_{\text {tot }} \approx 17.5 \mathrm{mag}$ (Vega relative), 11 sources are detected, nine of which are extragalactic. The integrated galaxy number counts are therefore $\sim 1.3 \times 10^{4} \mathrm{deg}^{-2}$ to this depth. The galaxies detected at $3.2 \mu \mathrm{m}$ have a median redshift of $z=0.56$. All $3.2 \mu \mathrm{m}$ sources have $1.6 \mu \mathrm{m}, 1.1 \mu \mathrm{m}$, and visual counterparts, all of fairly regular morphology; several also have $6.7 \mu \mathrm{m}, 15 \mu \mathrm{m}, 850 \mu \mathrm{m}, 8.5 \mathrm{GHz}$, or $1.4 \mathrm{GHz}$ counterparts. No sources are found that are either anomalously red or anomalously blue in their $H-[3.2]$ color, and there are significant detections of most of the known near-infrared HDF sources for which detection in these $3.2 \mu \mathrm{m}$ data seemed likely.

Key words: galaxies: photometry - galaxies: statistics - infrared radiation - techniques: photometric
\end{abstract}

\section{INTRODUCTION}

Until now, it has been impractical to make wide-field ground-based surveys at infrared wavelengths longer than $2.4 \mu \mathrm{m}$. Although the atmosphere is transparent in wavelength windows in the near-infrared out to $10 \mu \mathrm{m}$, sky brightness increases rapidly with increasing wavelength. Detector area is limited by the need to read out quickly before saturation. The Near Infrared Camera (NIRC; Matthews \& Soifer 1994) on the $10 \mathrm{~m} \mathrm{W.} \mathrm{M.} \mathrm{Keck} \mathrm{Telescope,}$ however, is equipped with a fast readout mode that allows it to read out its entire array quickly enough to avoid saturation in a short-wavelength $L$-band filter, centered on $3.2 \mu \mathrm{m}$.

The $3.2 \mu \mathrm{m}$ bandpass is complementary to the standard near-infrared $J, H$, and $K(1.2,1.6$, and $2.2 \mu \mathrm{m})$ bandpasses in a number of respects. It admits light at the rest-frame wavelengths of these bandpasses at redshifts of 1.7, 1.0, and 0.5 , so observations of intermediate- and high-redshift galaxies can be compared directly with local analogs observed in the standard bandpasses. The $3.2 \mu \mathrm{m}$ bandpass can be used to extend the near-infrared spectral energy distributions (SEDs) to longer wavelengths. While stellar populations are made up of a superposition of basically thermal radiators, nuclear activity tends to be redder than thermal (in the near-infrared; bluer in the visual) and thus ought to become more prominent in redder bandpasses. Nuclear activity may also heat nuclear dust to produce additional near-infrared emission (see, e.g., Peletier et al. 1999). In units of $f_{v}$, the SEDs of normal galaxies peak at $\approx 1.6 \mu \mathrm{m}$, primarily as a result of a minimum in $\mathrm{H}^{-}$opacity in stellar atmospheres. At $z=1$, this peak is redshifted to $3.2 \mu \mathrm{m}$. A "nonevolving" $L^{*}$ elliptical galaxy at redshift $z=1$ ought

\footnotetext{
${ }^{1}$ Based on observations made at the W. M. Keck Observatory, which is operated jointly by the California Institute of Technology and the University of California, and with the NASA/ESA Hubble Space Telescope, which is operated by the Association of Universities for Research in Astronomy (AURA), Inc., under NASA contract NAS 5-26555.

${ }^{2}$ Institute for Advanced Study, Olden Lane, Princeton, NJ 08540; hogg@ias.edu.

${ }^{3}$ Hubble Fellow.

${ }^{4}$ Palomar Observatory, California Institute of Technology, Mail Stop 105-24, Pasadena, CA 91125.

${ }^{5}$ Space Telescope Science Institute, 3700 San Martin Drive, Baltimore, MD 21218.
}

to appear as a $\sim 17.5$ mag source at $3.2 \mu \mathrm{m}$. At $z=1$, old stellar populations are 1.5 to $3 \mathrm{mag}$ brighter than young stellar populations with the same apparent $V$ magnitude (Coleman, Wu, \& Weedman 1980; Aaronson 1977).

This paper presents the results of deep imaging of the Hubble Deep Field (HDF; Williams et al. 1996) in the 3.2 $\mu \mathrm{m}$ bandpass with NIRC. Although neither the sky coverage nor the number of detected sources is large, to our knowledge this is the first deep near-infrared survey at wavelengths beyond the $K$ band. It extends the SEDs of HDF sources over a decade in wavelength from the F300W bandpass at $0.3 \mu \mathrm{m}$. The choice of the HDF for deep imaging in this new $3.2 \mu \mathrm{m}$ bandpass was clear: the HDF is currently the most well studied field on the sky, deeply imaged by almost every astronomical technique.

In what follows, all magnitudes are Vega relative.

\section{OBSERVATIONS AND REDUCTION}

The $L_{\text {short }}$ filter on NIRC was chosen for this study. The filter passes light in the wavelength range $2.5 \mu \mathrm{m}<\lambda<3.5$ $\mu \mathrm{m}$. When combined with typical atmospheric absorption conditions on Mauna Kea, the bandpass is concentrated in the wavelength range $2.9 \mu \mathrm{m}<\lambda<3.5 \mu \mathrm{m}$, with a small "blue leak" ( $\sim 10 \%$ of the total transmission function $)$ in a narrow peak at $2.5 \mu \mathrm{m}$. The central wavelength is $\sim 3.2 \mu \mathrm{m}$ for the filter plus atmospheric transmission function; for this reason, magnitudes with this instrument and filter setup will be denoted [3.2]. The $L_{\text {short }}$ filter is currently the longest wavelength bandpass in which NIRC can read out its full $256 \times 256$ pixel $\left(38 \times 38 \operatorname{arcsec}^{2}\right)$ field area without saturation from sky brightness alone. A source with [3.2] $=0 \mathrm{mag}$ in the $L_{\text {short }}$ bandpass has a flux density of $330 \mathrm{Jy}$. This approximate zero point is an interpolation between measured $K$ - and $L$-band zero points and is uncertain by no more than $10 \%$.

The selection of the HDF for deep study with the Hubble Space Telescope (HST) is described elsewhere (Williams et al. 1996). Within the $H S T$-imaged portion of the HDF, a set of subfields were chosen for deep study with NIRC. These subfields were not chosen with regard to any particular "interesting sources." Rather, one subfield was chosen to lie roughly in the center of each Wide Field Planetary Camera 2 (WFPC2) CCD chip image, subject to guide-star constraints (these are the primary subfields A, B, C, and D), and 
additional subfields were chosen to fill in the gaps between these four primary subfields. In the end, telescope time and weather restricted our study to five subfields, the primary four and subfield F, which lies between subfields C and D. The well-observed solid angle is roughly $0.5 \mathrm{arcmin}^{2}$ per subfield (given dithering), or a total of $2.5 \mathrm{arcmin}^{2}$. The layout of the subfields is shown in Figure 1, relative to the $H S T$-imaged field.

Air mass of the observations ranged from 1.40 to 1.75 . The conditions were clear and apparently photometric for all nights, although there was some variation in the sky brightness. Each individual exposure consisted of 1000 (or, occasionally, 2000) 0.025 or $0.029 \mathrm{~s}$ integrations co-added into a single image in hardware. A visual-imaging offset guider pointing at a star maintained the tracking of the telescope. The observations were taken in half-hour or hour-long sets with random dithers over a $10 \times 10 \operatorname{arcsec}^{2}$ range. The photometric sensitivity was determined by measuring faint standard stars (Elias et al. 1982; Persson et al. 1998) roughly once per hour during each night. During several of the nights, the measured sensitivity (corrected for air mass) varied in an apparently linear way with time. The subfield most affected by this was subfield A, which shows no significant sources (see below), so the variation in this subfield does not affect the results. The scatter of the individual standard-star measurements around the linear sensitivity trends is $\pm 0.05 \mathrm{mag}$, but for the other four subfields, the trends themselves range between less than $\pm 0.01 \mathrm{mag}$ for subfield $D$ and \pm 0.12 mag for subfield $F$. The uncertainty in the photometric calibration is thus no better than $0.05 \mathrm{mag}$, but it may be worse if the linear trends represent a serious photometric problem. The observing dates, integration times, and sensitivities of the final, stacked images of the subfields are given in Table 1.

The data were processed by subtracting an average of the two adjacent images (before and after) from each image to eliminate artifacts introduced by varying sky brightness and field rotation resulting from the alt-azimuth configuration of the Keck Telescope. Because the individual images show no significant sources above the sky noise, registration of the individual images was accomplished with telescope coordinates as computed from the positions of the star in the guider camera. The individual registered images were averaged, after dark subtraction and flat-fielding, to

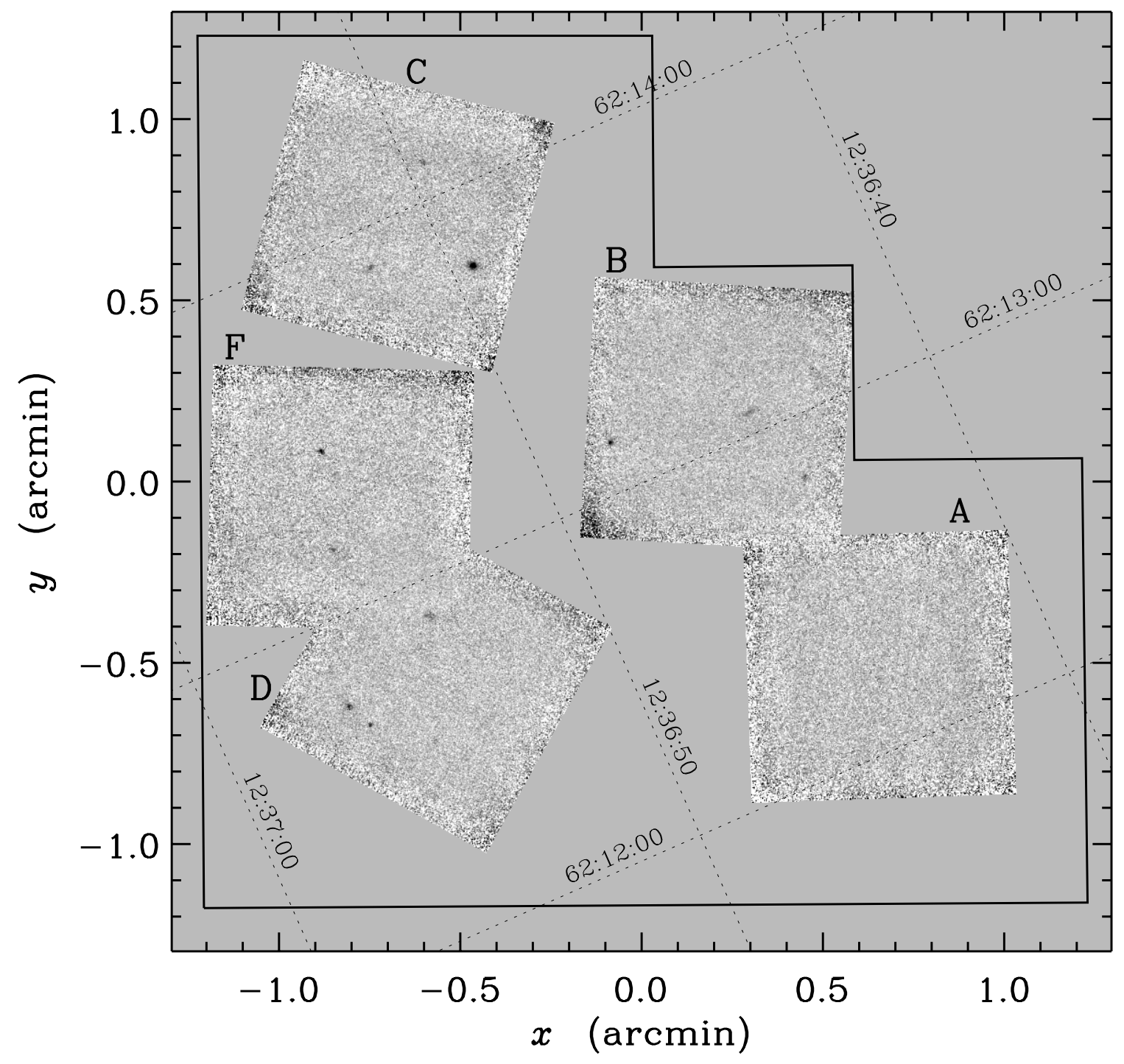

FIG. 1.-Final $3.2 \mu \mathrm{m}$ mosaic. The subfields are labeled with letters. The outline of the HST WFPC2-imaged field is shown with a solid line. Lines of constant right ascension and declination are shown and labeled. 
TABLE 1

Properties of the Final, Stacked Images of the Subfields

\begin{tabular}{ccccc}
\hline \hline Name & \multicolumn{1}{c}{$\begin{array}{c}\text { Date } \\
(\mathrm{UT})\end{array}$} & $\begin{array}{c}\text { Exposure } \\
(\mathrm{s})\end{array}$ & $\begin{array}{c}\text { Seeing FWHM } \\
(\operatorname{arcsec})\end{array}$ & $\begin{array}{c}{[3.2] \text { at } 1 \sigma^{\mathrm{b}}} \\
(\mathrm{mag})\end{array}$ \\
\hline NIRC-HDF-A ...... & 1999 Apr 28 & 13440 & $0.5^{\mathrm{c}}$ & 20.1 \\
NIRC-HDF-B ...... & 1998 Feb 6 & 11100 & 0.5 & 20.4 \\
NIRC-HDF-C ....... & 1998 Feb 7 & 9600 & 0.4 & 20.2 \\
NIRC-HDF-D ...... & 1999 Apr 30 & 9480 & 0.4 & 20.3 \\
NIRC-HDF-F ...... & 1999 Apr 27 & 11100 & 0.6 & 20.0 \\
\hline
\end{tabular}

${ }^{a}$ Because there are so few significant sources per subfield, the seeing measurements are very uncertain; see text.

${ }^{\mathrm{b}}$ The $1 \sigma$ magnitude is that corresponding to a $1 \sigma$ variation in the sky in a focal-plane aperture of $1 \operatorname{arcsec}^{2}$ in solid angle, in the central (i.e., full exposure time) part of the image.

c The seeing FWHM for subfield A is taken from the observations of the standard stars, because there are no significant sources detected in that subfield.

TABLE 2

The Catalog Selected at $3.2 \mu \mathrm{m}$

\begin{tabular}{ccccc}
\hline \hline $\begin{array}{c}\text { R.A. }^{\mathrm{a}} \\
(\mathrm{J} 2000.0)\end{array}$ & $\begin{array}{c}\text { Decl. }^{\mathrm{a}} \\
(\mathrm{J} 2000.0)\end{array}$ & Subfield & $\begin{array}{c}{[3.2]_{\mathrm{ap}}^{\mathrm{b}}} \\
(\mathrm{mag})\end{array}$ & $\begin{array}{c}{[3.2]_{\mathrm{tot}}{ }^{\mathrm{c}}} \\
(\mathrm{mag})\end{array}$ \\
\hline $123644.017 \ldots \ldots$ & +621250.11 & $\mathrm{~B}$ & $17.25 \pm 0.15$ & 16.70 \\
$123644.626 \ldots \ldots$ & +621304.29 & $\mathrm{~B}$ & $17.46 \pm 0.14$ & 16.90 \\
$123648.088 \ldots \ldots$ & +621309.21 & $\mathrm{~B}$ & $16.78 \pm 0.10$ & 16.51 \\
$123649.435 \ldots \ldots$ & +621346.92 & $\mathrm{C}$ & $15.75 \pm 0.04$ & 15.44 \\
$123649.541 \ldots \ldots$. & +621406.85 & $\mathrm{C}$ & $17.57 \pm 0.18$ & 17.43 \\
$123651.783 \ldots \ldots$. & +621353.85 & $\mathrm{C}$ & $17.25 \pm 0.15$ & 16.99 \\
$123653.916 \ldots \ldots$ & +621254.26 & $\mathrm{D}$ & $17.11 \pm 0.11$ & 16.84 \\
$123654.742 \ldots \ldots$. & +621328.01 & $\mathrm{~F}$ & $16.80 \pm 0.11$ & 16.71 \\
$123655.460 \ldots \ldots$ & +621311.63 & $\mathrm{~F}$ & $17.40 \pm 0.18$ & 17.07 \\
$123656.368 \ldots \ldots$. & +621241.14 & $\mathrm{D}$ & $17.80 \pm 0.21$ & 17.72 \\
$123656.675 \ldots \ldots$ & +621245.51 & $\mathrm{D}$ & $16.85 \pm 0.09$ & 16.19 \\
\hline
\end{tabular}

NotE.- Units of right ascension are hours, minutes, and seconds, and units of declination are degrees, arcminutes, and arcseconds.

${ }^{\text {a }}$ Absolute positions were found with information from the HST WFPC2 images (Williams et al. 1996).

${ }^{b}$ Aperture magnitudes [3.2] $]_{\mathrm{ap}}$ are measured in a 2".0 diameter focal-plane aperture. The quoted uncertainty in magnitude is simply that due to uncertainty in the photon statistics and sky level in the focal-plane aperture. The quoted uncertainty does not take into account uncertainty in overall calibration.

c Total magnitudes [3.2 $]_{\text {tot }}$ have been corrected to an effective 6".0 diameter aperture with information from the $H$ image; see text for details.

TABLE 3

REDSHIFTS AND COLORS OF THE $3.2 \mu \mathrm{m}$ SOURCES

\begin{tabular}{cccccccccc}
\hline \hline $\begin{array}{c}\text { R.A. } \\
(\mathrm{J} 2000.0)\end{array}$ & $\begin{array}{c}\text { Decl. } \\
(\mathrm{J} 2000.0)\end{array}$ & $\begin{array}{c}{[3.2]_{\mathrm{tot}}} \\
(\mathrm{mag})\end{array}$ & $z^{\mathrm{a}}$ & $\begin{array}{c}U-B^{\mathrm{b}} \\
(\mathrm{mag})\end{array}$ & $\begin{array}{c}B-V^{\mathrm{b}} \\
(\mathrm{mag})\end{array}$ & $\begin{array}{c}V-I^{\mathrm{b}} \\
(\mathrm{mag})\end{array}$ & $\begin{array}{c}I-J^{\mathrm{b}} \\
(\mathrm{mag})\end{array}$ & $\begin{array}{c}J-H^{\mathrm{b}} \\
(\mathrm{mag})\end{array}$ & $\begin{array}{c}H-[3.2]^{\mathrm{b}, \mathrm{c}} \\
(\mathrm{mag})\end{array}$ \\
\hline $123644.017 \ldots \ldots$. & +621250.11 & 16.70 & 0.557 & -0.17 & 1.26 & 1.15 & 0.66 & 1.26 & 1.78 \\
$123644.626 \ldots \ldots$ & +621304.29 & 16.90 & 0.485 & 0.28 & 1.58 & 1.29 & 0.81 & 1.29 & 1.78 \\
$123648.088 \ldots \ldots$. & +621309.21 & 16.51 & 0.476 & 1.22 & 1.93 & 1.38 & 0.67 & 1.23 & 1.65 \\
$123649.435 \ldots \ldots$. & +621346.92 & 15.44 & 0.089 & 1.46 & 1.19 & 0.95 & 0.56 & 0.99 & 0.81 \\
$123649.541 \ldots \ldots$. & +621406.85 & 17.43 & 0.752 & -0.82 & 0.96 & 1.34 & 0.67 & 1.24 & 2.01 \\
$123651.783 \ldots \ldots$. & +621353.85 & 16.99 & 0.557 & -0.34 & 1.21 & 1.21 & 0.69 & 1.28 & 1.73 \\
$123653.916 \ldots \ldots$. & +621254.26 & 16.84 & 0.642 & -0.55 & 1.12 & 1.31 & 0.68 & 1.26 & 1.72 \\
$123654.742 \ldots \ldots$. & +621328.01 & 16.71 & 0.000 & 2.43 & 1.49 & 1.19 & 0.65 & 0.89 & 0.58 \\
$123655.460 \ldots \ldots$ & +621311.63 & 17.07 & 0.968 & 73.64 & 2.26 & 2.08 & 1.26 & 1.37 & 1.98 \\
$123656.368 \ldots \ldots$. & +621241.14 & 17.72 & 0.000 & 0.76 & 0.49 & 0.70 & 0.48 & 0.61 & -0.04 \\
$123656.675 \ldots \ldots$. & +621245.51 & 16.19 & 0.518 & 1.57 & 2.04 & 1.60 & 0.83 & 1.29 & 1.63 \\
\hline
\end{tabular}

a Redshifts are from Cohen et al. 1996, Lowenthal et al. 1997, and Cohen et al. 1999. Sources with "0.000" in this column are Galactic stars.

${ }^{b}$ Colors were measured through 2".0 diameter apertures in images smoothed to match the seeing of the $3.2 \mu \mathrm{m}$ images; see text. The bandpass names $(U, B, V, I, J, H)$ stand for F300W, F450W, F606W, F814W, F110W, and F160W. Uncertainties in all colors except $U-B$ and $H-$ [3.2] are at or below the $0.01 \mathrm{mag}$ level; see text

${ }^{c}$ Uncertainties in $H-[3.2]$ color are dominated by the uncertainty in the [3.2] $]_{\mathrm{ap}}$ magnitude. 

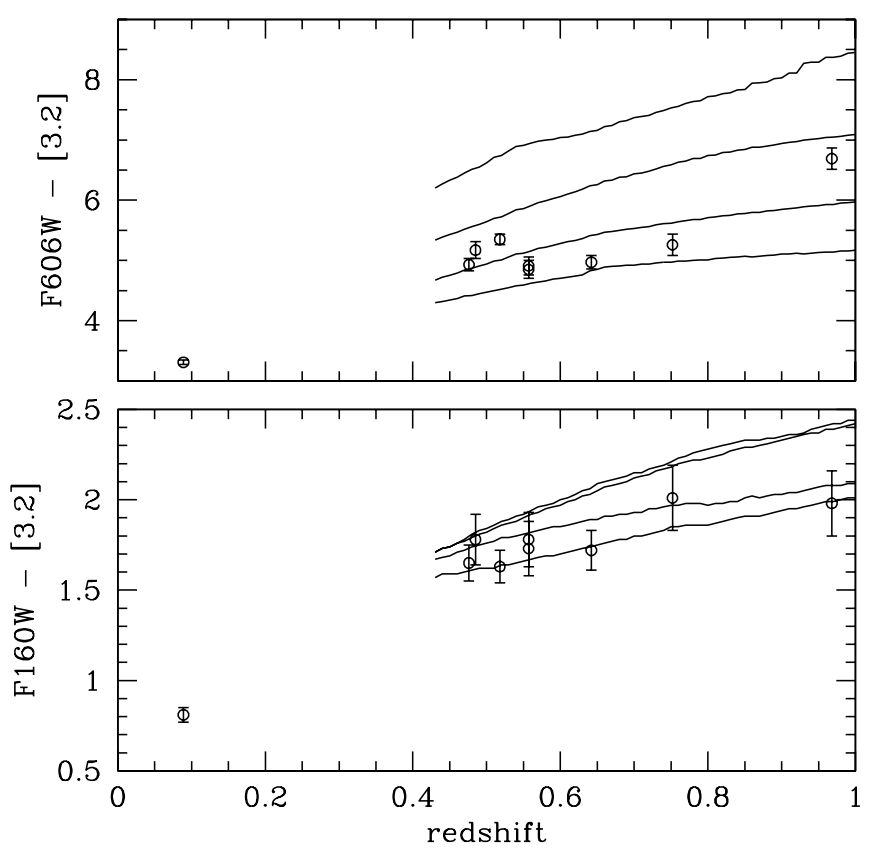

FIG. 2.-The $V-[3.2]$ and $H-$ [3.2] colors as a function of redshift for the sources selected at $3.2 \mu \mathrm{m}$. Also shown are no-evolution predictions for galaxies of types (from reddest to bluest, or top to bottom) E/S0, Sbc, Scd, and $\mathrm{Im}$, based on the bright-galaxy samples of Coleman, Wu, \& Weedman (1980) and Aaronson (1977). The predicted color-redshift tracks begin at redshift $z=0.43$ because the bright-galaxy data do not extend longward of the $K$ band.

produce the final images shown in Figure 1. The pixel-topixel rms values in the central parts of the images correspond to $\approx 20.2 \mathrm{mag}$ in $1 \operatorname{arcsec}^{2}$; the specific values for each subfield are given in Table 1.

Because there are so few sources visible per subfield (none for subfield A), determination of the seeing is difficult. Seeing values given in Table 1 are determined by comparison of the sources in the final, stacked images with their visual counterparts in the WFPC2 image of the HDF taken in the F814W bandpass (Williams et al. 1996); they are necessarily quite uncertain. Since in several fields (especially F) the seeing in the final, stacked image is apparently worse than that for the standard stars, some of the seeing must arise from poor registration of the frames co-added in the stacks.

For visual images, the "version 2" reductions of the $H S T$ WFPC2 imaging of the HDF were used. Details of the data acquisition, reduction, and sensitivities are given elsewhere (Williams et al. 1996). For brevity, the F300W, F450W, F606W, and F814W bandpasses will be referred to here as $U, B, V$, and $I$, although $\mathrm{F} 300 \mathrm{~W}$ and $\mathrm{F} 606 \mathrm{~W}$ are substantially different from conventional $U$ and $V$.

For other near-infrared data, the "version 1.04 " reductions of the imaging of the HDF taken with the NICMOS instrument on HST were used. Details of the data acquisition, reduction, and sensitivities are given elsewhere (Dickinson et al. 2000). For brevity, the F110W and F160W bandpasses will be referred to here as $J$ and $H$, although F110W is substantially bluer than standard $J$. The photometric sensitivities of the $J$ and $H$ data are uncertain at the $\sim 5 \%$ level.

\section{SOURCE SELECTION AND PHOTOMETRY}

Sources were identified in the $3.2 \mu \mathrm{m}$ image by requiring a positive deviation of greater significance than $5.0 \sigma$ inside a focal-plane aperture of 1".0 diameter in the final, stacked images of the subfields. The $1 \sigma$ noise levels were estimated by measuring fluxes in randomly placed apertures, so they correctly take into account any pixel-to-pixel correlations. At significances less than $10 \sigma$, it was established that the sources had visual and near-infrared counterparts in the $H S T$ images. No $5.0 \sigma$ deviations failed to show counterparts, except at the very edges of the subfields, where the noise and background flatness are degraded. A total of 11 sources were selected. The total area subject to source detection is roughly $2.5 \mathrm{arcmin}^{2}$.

Total magnitudes [3.2] $]_{\text {tot }}$ for the sources selected at 3.2 $\mu \mathrm{m}$ were estimated by measuring aperture magnitudes $[3.2]_{\mathrm{ap}}$ in focal-plane apertures of 2".0 diameter and then correcting for the small size of the aperture. The 2".0 aperture was chosen to be small enough to reduce the background at $3.2 \mu \mathrm{m}$ but large enough to reduce variations in photometric sensitivity measured from the standard stars to below $0.1 \mathrm{mag}$. The aperture corrections were determined under the assumption that there are only small color gradients in $H-$ [3.2] color, by comparing 2."0 and 6".0 diameter aperture magnitudes in the very high signal-to-noise ratio $H$-band image, smoothed to the seeing of the $3.2 \mu \mathrm{m}$ image. (The one exception is the star at $12^{\mathrm{h}} 36^{\mathrm{m}} 56^{\mathrm{s}} .368$, $+62^{\circ} 12^{\prime} 41^{\prime \prime} 14$ [J2000.0], for which a 5".0 diameter aperture was used in order to avoid light from the neighboring bright galaxy.) Aperture and total magnitudes for the sources are given in Table 2. The uncertainties given in Table 2 are based only on the photon statistics and the uncertainties in determining the sky level and do not include uncertainties in the photometric sensitivity. No estimate is made for the uncertainties in the aperture corrections. These may be considerable, if the seeing estimates are in error, if there are significant color gradients, or if there is significant light outside of the 6." 0 diameter aperture.

Colors were measured in 2".0 diameter focal-plane apertures in the visual WFPC2 and near-infrared NICMOS images, smoothed to the seeing of the $3.2 \mu \mathrm{m}$ images. The aperture size was chosen so the colors were measured in the regions of the sources that are well measured at $3.2 \mu \mathrm{m}$. The colors are given in Table 3. The photon and read noise contributions to the uncertainties in the color measurements are at or below the $0.01 \mathrm{mag}$ level for all the visual and near-infrared colors except for $H-$ [3.2], where the uncertainties are dominated by the uncertainty in $[3.2]_{\mathrm{ap}}$ given in Table 2, and $U-B$, where the uncertainties are at the 0.01 to $0.2 \mathrm{mag}$ level, depending on $U$-band flux. Although the NICMOS $J$ and $H$ measurements have a very high signal-to-noise ratio, the photometric sensitivities are uncertain at the $\sim 10 \%$ level (Dickinson et al. 2000). Figure 2 shows $V-[3.2]$ and $H-$ [3.2] colors for the galaxies as a function of redshift. Figure 3 shows the visual and nearinfrared counterparts of all the sources.

Absolute right ascension and declination positions were assigned to the sources under the assumption that the absolute astrometry of the visual HST images of the HDF (Williams et al. 1996) is correct. These absolute positions are given in Table 2.

\section{COMPARISON WITH OTHER HDF CATALOGS}

The catalog selected at $3.2 \mu \mathrm{m}$ can be compared with other catalogs made in the HDF at other wavelengths or with other techniques. The redshifts and flux densities at other wavelengths discussed in this section are presented in Tables 3 and 4. 


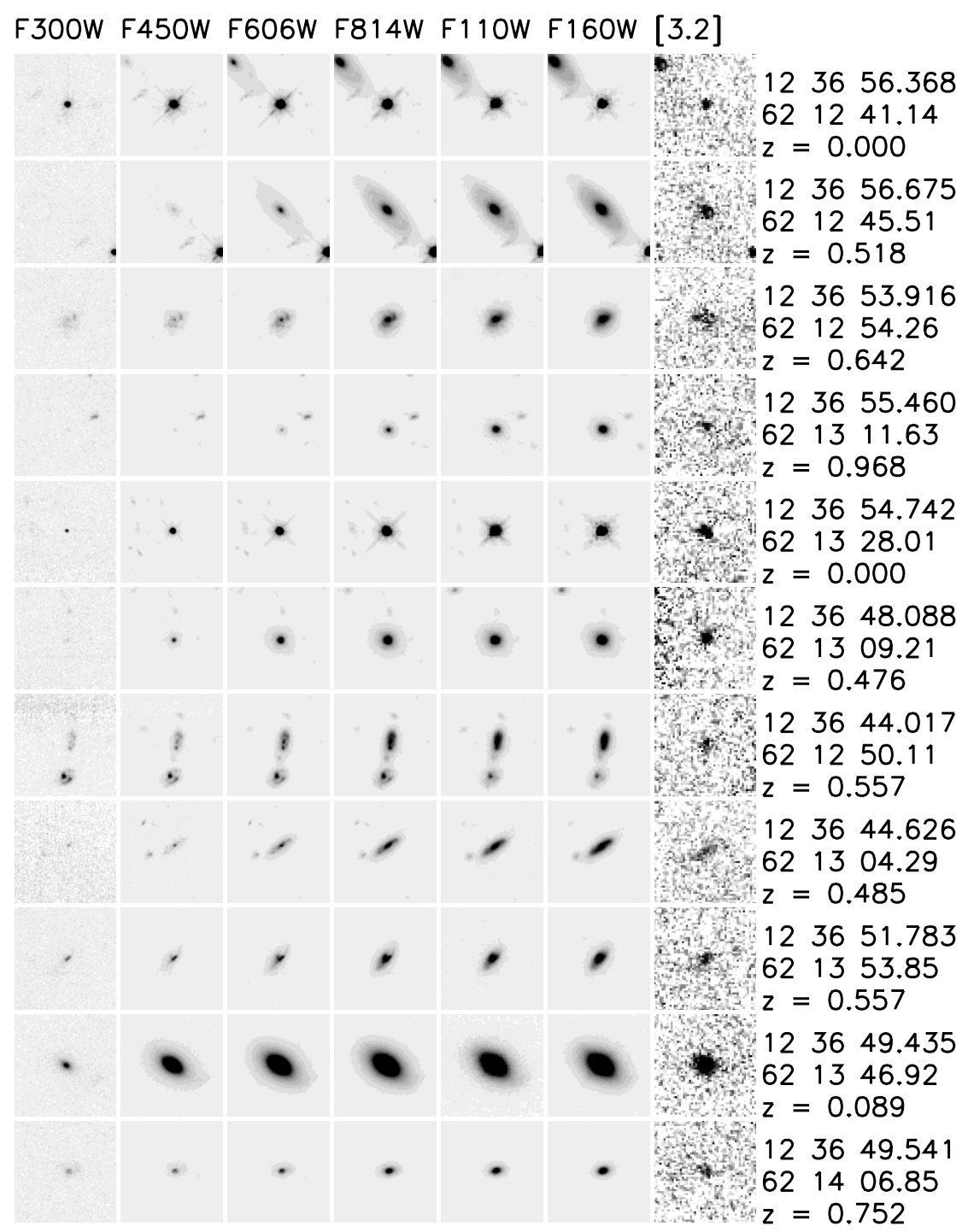

Fig. 3.-The $2^{\prime \prime} \times 2^{\prime \prime}$ region around each source selected at $3.2 \mu \mathrm{m}$. The images are stretched identically in terms of flux density per logarithmic interval in frequency $\left(v f_{v}\right)$.

TABLE 4

COUNTERParts TO THE EXTRAgalactic $3.2 \mu \mathrm{m}$ Sources

\begin{tabular}{|c|c|c|c|c|c|c|c|c|c|}
\hline $\begin{array}{c}\text { R.A. } \\
(\mathrm{J} 2000.0)\end{array}$ & $\begin{array}{c}\text { Decl. } \\
\text { (J2000.0) }\end{array}$ & $z$ & $\begin{array}{c}2.15 \mu \mathrm{m}^{\mathrm{a}} \\
(\mu \mathrm{Jy})\end{array}$ & $\begin{array}{c}3.2 \mu \mathrm{m}^{\mathrm{b}} \\
(\mu \mathrm{Jy})\end{array}$ & $\begin{array}{c}6.7 \mu \mathrm{m}^{\mathrm{c}} \\
(\mu \mathrm{Jy})\end{array}$ & $\begin{array}{c}15 \mu \mathrm{m}^{\mathrm{c}} \\
(\mu \mathrm{Jy})\end{array}$ & $\begin{array}{c}850 \mu \mathrm{m}^{\mathrm{d}} \\
(\mu \mathrm{Jy})\end{array}$ & $\begin{array}{c}8.5 \mathrm{GHz}^{\mathrm{e}} \\
(\mu \mathrm{Jy})\end{array}$ & $\begin{array}{c}1.4 \mathrm{GHz}^{\mathrm{e}} \\
(\mu \mathrm{Jy})\end{array}$ \\
\hline $123644.017 \ldots \ldots$ & +621250.11 & 0.557 & $55.9 \pm 3.1$ & $69.2 \pm 9.6$ & $<50$ & $282_{-64}^{+60}$ & $\cdots$ & $10.2 \pm 1.8$ & $<23.0$ \\
\hline $123648.088 \ldots \ldots$ & +621309.21 & 0.476 & $74.3 \pm 2.7$ & $82.4 \pm 7.6$ & $\ldots$ & $\ldots$ & $\ldots$ & $\ldots$ & $\ldots$ \\
\hline $123649.435 \ldots \ldots$ & +621346.92 & 0.089 & $362.5 \pm 5.7$ & $220.8 \pm 8.1$ & $\dddot{41}_{-30}^{+66}$ & $<52$ & $\cdots$ & $\cdots$ & $\cdots$ \\
\hline $123649.541 \ldots \ldots$ & +621406.85 & 0.752 & $21.8 \pm 1.5$ & $35.3 \pm 5.9$ & $<40$ & $150_{-48}^{+74}$ & $\ldots$ & $\ldots$ & $\ldots$ \\
\hline $123655.460 \ldots \ldots$ & +621311.63 & 0.968 & $39.4 \pm 2.4$ & $49.2 \pm 8.2$ & $<37$ & $23_{-11}^{+10}$ & $\ldots$ & $12.3 \pm 1.8$ & $<23.0$ \\
\hline $123656.675 \ldots \ldots$ & +621245.51 & 0.518 & $109.5 \pm 4.1$ & $110.7 \pm 9.2$ & $\ldots$ & $\ldots$ & $\ldots$ & $\ldots$ & $\ldots$ \\
\hline
\end{tabular}

${ }^{a}$ Flux densities at $2.15 \mu \mathrm{m}$ are from Hogg et al. 2000, converted to janskys under the assumption that $K_{s}=0$ mag corresponds to $710 \mathrm{Jy}$.

${ }^{\mathrm{b}}$ Flux densities at $3.2 \mu \mathrm{m}$ are [3.2] $]_{\text {tot }}$ magnitudes converted under the assumption that [3.2] $=0$ mag corresponds to $330 \mathrm{Jy}$.

${ }^{c}$ Flux densities at 6.7 and $15 \mu \mathrm{m}$ are from Aussel et al. 1999.

${ }^{d}$ Flux density at $850 \mu \mathrm{m}$ is from Hughes et al. 1998. The value is marked with a question mark because there is more than one possible $1.4 \mathrm{GHz}$ counterpart.

${ }^{\text {e }}$ Flux densities at 8.5 and $1.4 \mathrm{GHz}$ are from Richards et al. 1998 and Richards 2000. 
Redshifts were found by comparing the coordinates of the sources with the lists of redshifts obtained in the HDF with the Keck Telescope (Cohen et al. 1996; Lowenthal et al. 1997; Cohen et al. 2000). Two of the sources are Galactic stars, consistent with their stellar appearance on the $H S T$ images.

Flux densities in the $K_{s}$ band $(2.15 \mu \mathrm{m})$ were found by matching the absolute positions of the $3.2 \mu \mathrm{m}$ sources with the positions of the sources from the $K_{s}$-selected catalog of Hogg et al. (2000). Flux densities at 6.7 and $15 \mu \mathrm{m}$ were found by matching with the catalogs made from imaging data from the Infrared Space Observatory (Aussel et al. 1999; based on data from Serjeant et al. 1997). The flux density at $850 \mu \mathrm{m}$ was taken from the catalog made using the SCUBA instrument at the James Clerk Maxwell Telescope (Hughes et al. 1998), although the source was identified in part through comparing positions derived from 1.4 $\mathrm{GHz}$ observations (Richards 1999). Flux densities at 8.5 and $1.4 \mathrm{GHz}$ were found by matching positions in catalogs made with VLA data at these frequencies (Richards et al. 1998; Richards 2000).

The detection fraction at $3.2 \mu \mathrm{m}$ of known near-infrared sources was tested by identifying sources in the NICMOS HDF catalogs (Dickinson et al. 2000) with predicted $3.2 \mu \mathrm{m}$ magnitudes satisfying

$$
[3.2]_{\text {pred }}=\mathrm{F} 160 \mathrm{~W}-1.85(\mathrm{~F} 110 \mathrm{~W}-\mathrm{F} 160 \mathrm{~W})+0.25<18 \text {, }
$$

which corresponds to an approximate linear extrapolation (in log-log space) of the $J$ and $H$ magnitudes to [3.2]. (This equation is roughly appropriate for predicting the fluxes of galaxies and nuclear emission, since both have SEDs that resemble power laws at $3.2 \mu \mathrm{m}$ [observed] at the redshifts of interest.) All nine sources inside the region of the HDF well observed at $3.2 \mu \mathrm{m}$ with $[3.2]_{\text {pred }}<17.0$ have counterparts in the catalog selected at $3.2 \mu \mathrm{m}$, and of the six sources in the well-observed region with $17.0<[3.2]_{\text {pred }}<18.0$, two have counterparts in the catalog.

\section{RESULTS}

Images of the Hubble Deep Field at $3.2 \mu \mathrm{m}$, taken with the $10 \mathrm{~m}$ Keck Telescope, were presented, representing the first near-infrared survey of the distant universe at wavelengths beyond the $K$ band. The images cover a total area of $\sim 2.5 \operatorname{arcmin}^{2}$. To a $5 \sigma$ limit of $[3.2]_{\text {tot }} \approx 17.5 \mathrm{mag}$ (Vega relative), 11 sources are detected, nine of which are extragalactic. The integrated galaxy number counts are therefore $\sim 1.3 \times 10^{4} \mathrm{deg}^{-2}$ to this depth. The sources detected at 3.2 $\mu \mathrm{m}$ have a median redshift of $z=0.56$.

All $3.2 \mu \mathrm{m}$ sources have $1.6 \mu \mathrm{m}, 1.1 \mu \mathrm{m}$, and visual counterparts. No sources are found that are either anom- alously red or anomalously blue in their $V-[3.2]$ or $H-$ [3.2] color. In Figure 2, the source colors are compared with no-evolution predictions, based on observations of local galaxies (Coleman et al. 1980; Aaronson 1977) and simple models of the filter transmission curves. Although the sources selected at $3.2 \mu \mathrm{m}$ are among the reddest visually selected galaxies, they are blue relative to noevolution predictions. The blue colors are likely a consequence of higher star formation rates at intermediate redshift than in the present-day universe, as has been inferred, among other ways, from metallicity in Ly $\alpha$ clouds (Pei \& Fall 1995), ultraviolet luminosity density (Lilly et al. 1996; Connolly et al. 1997; Madau, Pozzetti, \& Dickinson 1998), and emission-line strengths (Hammer et al. 1997; Heyl et al. 1997; Small, Sargent, \& Hamilton 1997; Hogg et al. 1998). The lack of very red sources does not constrain the abundance of "extremely red objects" (EROs), because, at the magnitudes of interest, the angular number density of EROs is on the order of $10^{-2} \operatorname{arcmin}^{-2}$ (Hu \& Ridgway 1994; Thompson et al. 1999).

The morphologies of the $3.2 \mu \mathrm{m}$ sources in the visual and near-infrared bandpasses are shown in Figure 3. The sample is small, but they appear fairly regular, with some showing nearby neighbors or evidence for mild interaction.

Comparisons with surveys of the HDF at other wavelengths show that all of the $3.2 \mu \mathrm{m}$ sources have $2.15 \mu \mathrm{m}$ counterparts, one has a $6.7 \mu$ m counterpart, several have 15 $\mu \mathrm{m}$ counterparts, one has a likely $850 \mu \mathrm{m}$ counterpart, and two have $8.5 \mathrm{GHz}$ counterparts. Furthermore, there are 3.2 $\mu \mathrm{m}$ detections of the majority of sources that, on the basis of their $1.1 \mu \mathrm{m}$ and $1.6 \mu \mathrm{m}$ photometry, ought to have been detected.

In short, the $3.2 \mu \mathrm{m}$ imaging of the HDF shows no great surprises. It does demonstrate that it is possible, with significant telescope time, to perform studies of normal galaxies at high redshifts in the $L$ band of atmospheric transmission.

It is a pleasure to thank the $\mathrm{P} 3$ Team, in particular Al Conrad and Bob Goodrich, for the work on the NIRC instrument, which made this study possible, John Gathright for technical support, the HDF NICMOS General Observer team for providing near-infrared data in advance of publication, and Eric Richards for providing radio data in advance of publication. Financial support was provided by the NSF and by grants HF-01093.01-97A (Hubble Fellowship) and GO-07817.01-96A (NICMOS data) from the Space Telescope Science Institute, which is operated by AURA, Inc., under NASA contract NAS 5-26555. This research made use of the NASA ADS Abstract Service, the NOAO IRAF data reduction software, and the SM plotting software.

\section{REFERENCES}

Aaronson, M. 1977, Ph.D. thesis, Harvard Univ.

Aussel, H., Cesarsky, C. J., Elbaz, D., \& Starck, J. L. 1999, A\&A, 342, 313

Cohen, J. G., Cowie, L. L., Hogg, D. W., Songaila, A., Blandford, R., Hu, E. M., \& Shopbell, P. 1996, ApJ, 471, L5

Cohen, J. G., Hogg, D. W., Blandford, R., Cowie, L. L., Hu, E., Songaila, A., Shopbell, P., \& Richberg, K. 2000, ApJ, in press

Coleman, G. D., Wu, C.-C., \& Weedman, D. W. 1980, ApJS, 43, 393

Connolly, A. J., Szalay, A. S., Dickinson, M., SubbaRao, M. U., \& Brunner, R. J. 1997, ApJ, 486, L11

Dickinson, M. E., et al. 2000, in preparation

Elias, J. H., Frogel, J. A., Matthews, K., \& Neugebauer, G. 1982, AJ, 87, 1029
Hammer, F, et al. 1997, ApJ, 481, 49

Heyl, J., Colless, M., Ellis, R. S., \& Broadhurst, T. 1997, MNRAS, 285, 613 Hogg, D. W., Cohen, J. G., Blandford, R., \& Pahre, M. A. 1998, ApJ, 504, 622

Hogg, D. W., et al. 2000, ApJS, 127, 530

Hu, E. M., \& Ridgway, S. E. 1994, AJ, 107, 1303

Hughes, D. H., et al. 1998, Nature, 394, 241

Lilly, S. J., Le Fèvre, O., Hammer, F., \& Crampton, D. 1996, ApJ, 460, L1 Lowenthal, J. D., et al. 1997, ApJ, 481, 673

Madau, P., Pozzetti, L., \& Dickinson, M. 1998, ApJ, 498, 106

Matthews, K., \& Soifer, B. T. 1994, in Infrared Astronomy with Arrays:

The Next Generation, ed. I. S. McLean (Dordrecht: Kluwer), 239 
Pei, Y. C., \& Fall, S. M. 1995, ApJ, 454, 69

Peletier, R. F., Knapen, J. H., Shlosman, I., Pérez-Ramírez, D., Nadeau, D., Doyon, R., Rodriguez Espinosa, J. M., \& Pérez García, A. M. 1999, ApJS, 125, 363

Persson, S. E., Murphy, D. C., Krzeminski, W., Roth, M., \& Rieke, M. J. 1998, AJ, 116, 2475

Richards, E. A. 1999, ApJ, 513, L9
Richards, E. A. 2000, ApJ, in press

Richards, E. A., Kellerman, K. I., Fomalont, E. B., Windhorst, R. A., \& Partridge, R. B. 1998, AJ, 116, 1039

Serjeant, S. B. G., et al. 1997, MNRAS, 289, 457

Small, T. A., Sargent, W. L. W., \& Hamilton, D. 1997, ApJ, 487, 512

Thompson, D., et al. 1999, ApJ, 523, 100

Williams, R. E., et al. 1996, AJ, 112, 1335 